ARTICLE

Received 29 Oct 2013 | Accepted 28 Jan 2014 | Published 25 Feb $2014 \quad$ DOl: 10.1038/ncomms4333

\title{
Giant magnetic anisotropy and tunnelling of the magnetization in $\mathrm{Li}_{2}\left(\mathrm{Li}_{1-x} \mathrm{Fe}_{x}\right) \mathrm{N}$
}

\author{
A. Jesche ${ }^{1}$, R.W. McCallum¹,2, S. Thimmaiah¹, J.L. Jacobs¹,3, V. Taufour ${ }^{4}$, A. Kreyssig1,4, R.S. Houk ${ }^{1,3}$, \\ S.L. Bud'ko ${ }^{1,4} \&$ P.C. Canfield ${ }^{1,4}$
}

Large magnetic anisotropy and coercivity are key properties of functional magnetic materials and are generally associated with rare earth elements. Here we show an extreme, uniaxial magnetic anisotropy and the emergence of magnetic hysteresis in $\mathrm{Li}_{2}\left(\mathrm{Li}_{1}{ }_{x} \mathrm{Fe}_{x}\right) \mathrm{N}$. An extrapolated, magnetic anisotropy field of $220 \mathrm{~T}$ and a coercivity field of over $11 \mathrm{~T}$ at $2 \mathrm{~K}$ outperform all known hard ferromagnets and single-molecular magnets. Steps in the hysteresis loops and relaxation phenomena in striking similarity to single-molecular magnets are particularly pronounced for $x \ll 1$ and indicate the presence of nanoscale magnetic centres. Quantum tunnelling, in the form of temperature-independent relaxation and coercivity, deviation from Arrhenius behaviour and blocking of the relaxation, dominates the magnetic properties up to $10 \mathrm{~K}$. The simple crystal structure, the availability of large single crystals and the ability to vary the Fe concentration make $\mathrm{Li}_{2}\left(\mathrm{Li}_{1-x} \mathrm{Fe}_{x}\right) \mathrm{N}$ an ideal model system to study macroscopic quantum effects at elevated temperatures and also a basis for novel functional magnetic materials.

\footnotetext{
${ }^{1}$ The Ames Laboratory, lowa State University, Ames, lowa 50011, USA. ${ }^{2}$ Department of Materials Science and Engineering, lowa State University, Ames, lowa 50011, USA. ${ }^{3}$ Department of Chemistry, lowa State University, Ames, lowa 50011, USA. ${ }^{4}$ Department of Physics and Astronomy, lowa State University, Ames, lowa 50011, USA. Correspondence and requests for materials should be addressed to A.J. (email: jesche@ameslab.gov).
} 
C ontrolling individual spins on an atomic level is one of the major goals of solid-state physics and chemistry. To this effect, single-molecule magnets $(\mathrm{SMMs})^{1}$ have brought significant insight ranging from fundamental quantum effects like tunnelling of the magnetization ${ }^{2}$ and quantum decoherence ${ }^{3}$ to possible applications in quantum computing ${ }^{4}$ and high-density data storage ${ }^{5}$. Their basic magnetic units are coupled spins of a few magnetic atoms, which are embedded in and separated by complex organic structures. Whereas these are small magnetic units, they are still finite in extent and need to carefully balance coupling between magnetic atoms and isolation of one molecule from the next. Dimers of transition metals, as the small-size end point of SMMs, have been theoretically proposed to be promising candidates for novel information storage devices ${ }^{6}$. An alternative approach for the design of magnetic materials based on a few or even single atoms as magnetic units is ad-atoms on metallic surfaces $^{7,8}$. The key property among these actually very different examples, and of any nanoscale magnetic system, is a large magnetic anisotropy energy.

Basic magnetic units of SMMs are transition metal ion clusters $^{9}$, lanthanide ion clusters ${ }^{10,11}$ or mixed clusters of both $^{12}$. Even mononuclear complexes based on a single lanthanide ion have been realized ${ }^{13,14}$. The lanthanide-based systems are promising due to their large single-ion anisotropy $^{15,16}$, which often leads to large magnetic anisotropy energies. In contrast, single transition metal ions are seldom considered as suitable candidates and the number of reported attempts to use them as mononuclear magnetic units is limited ${ }^{17-19}$. The main reason is the widely known paradigm of 'orbital quenching'. This suppression of the orbital contribution to the magnetic moment by the crystal electric field leads to a comparatively small anisotropy energy (neglecting spin-orbit coupling, a pure spin contribution is by default isotropic). The absence of an orbital contribution is reflected, for example, in the largely isotropic magnetization of the elemental ferromagnets $\mathrm{Fe}$, $\mathrm{Co}$ and $\mathrm{Ni}^{20,21}$. However, Klatyk et al. ${ }^{22}$ have suggested that a rare interplay of crystal electric field effects and spin-orbit coupling causes a large orbital contribution to the magnetic moment of $\mathrm{Fe}$ in polycrystalline $\mathrm{Li}_{2}\left(\mathrm{Li}_{1-x} \mathrm{Fe}_{x}\right) \mathrm{N}$. On the basis of the strong increase of the magnetization on cooling 22 and on Mössbauer spectroscopy ${ }^{22,23}$, a ferromagnetic ordering with $T_{\mathrm{C}} \approx 65 \mathrm{~K}$ was inferred for $x=0.21$ and, furthermore, huge hyperfine fields were found. The orbital contribution to the magnetic moment of $\mathrm{Fe}$ as well as the large hyperfine fields were theoretically described within the framework of local density approximation (LDA) calculations ${ }^{22,24}$. Furthermore, a large magnetic anisotropy has been theoretically proposed ${ }^{22,24}$.

Here we show the experimental verification of the large anisotropy by magnetization measurements on single crystals and reveal a huge magnetic hysteresis as the key property of functional magnetic materials. More importantly, we have discovered that this highly anisotropic transition metal system manifests strong indications of a macroscopic quantum tunnelling of the magnetization in the form of pronounced steps in the magnetization loops and a temperature-independent relaxation. Tunnelling of the magnetization explicitly refers to the macroscopic tunnelling of the total magnetization and not to microscopic tunnelling events influencing the domain-wall movement in ferromagnets ${ }^{25}$. The $\mathrm{Li}_{3} \mathrm{~N}$ host provides an extremely anisotropic ligand field for the Fe atoms as well as an insulating environment in analogy to the 'organic framework' of SMMs. There are no indications for meso- or macroscopic phase separation (Supplementary Note 1). Although we cannot completely rule out cluster formation (for example, dimers or trimers of $\mathrm{Fe}$ on adjacent $\mathrm{Li}$ sites), the preponderance of the data supports a single iron atom as mononuclear magnetic centre, which is, furthermore, the simplest model and based on the fewest assumptions. The phenomenological similarities to SMMs indicate that the spontaneous magnetization and hysteresis are primarily caused by the extreme magnetic anisotropy and not by collective ordering phenomena. In accordance with earlier work on mononuclear systems, $\mathrm{Li}_{2}\left(\mathrm{Li}_{1-x} \mathrm{Fe}_{x}\right) \mathrm{N}$ might be considered as 'atomic magnet' ${ }^{26}$ or 'single-ionic SMM'15. The magnetic anisotropy, coercivity and energy barrier for spin inversion found in $\mathrm{Li}_{2}\left(\mathrm{Li}_{1-x} \mathrm{Fe}_{x}\right) \mathrm{N}$ are roughly one order of magnitude larger than in typical SMMs. Magnetic hysteresis exists up to comparatively high temperatures of $\mathrm{T} \gtrsim 16 \mathrm{~K}$ for $x \ll 1$ and is further enhanced up to $\mathrm{T} \gtrsim 50 \mathrm{~K}$ for the largest Fe concentration of $x=0.28$.

\section{Results}

Basic properties of $\mathbf{L i}_{2}\left(\mathrm{Li}_{1-x} \mathrm{Fe}_{x}\right) \mathbf{N}$. We grew $\mathrm{Li}_{2}\left(\mathrm{Li}_{1-x} \mathrm{Fe}_{x}\right) \mathrm{N}$ single crystals of several millimetre size (Fig. 1a,b) and Fe concentrations ranging over three orders of magnitude $x=0.00028$ to 0.28 by using a Li-flux method to create a rare, nitrogen-bearing metallic solution. $\mathrm{Li}_{2}\left(\mathrm{Li}_{1-x} \mathrm{Fe}_{x}\right) \mathrm{N}$ crystallizes in a hexagonal lattice, space group $P 6 / \mathrm{m} \mathrm{m} \mathrm{m}$, with a rather simple unit cell (Fig. 1c) and lattice parameters of $\mathrm{a}=3.652(8) \AA$ and $\mathrm{c}=3.870(10) \AA$ for $x=0$. Fe substitution causes an increase of $a$ but a decrease of $c$ by $1.1 \%$ and $1.5 \%$, respectively, for $x=0.28$ with intermediate concentrations showing a linear dependence on $x$ following Vegards law (Supplementary Fig. 1). As indicated by the notation of the chemical formula, the substituted $\mathrm{Fe}$ atoms occupy only the $\mathrm{Li}-1 b$ Wyckoff position, which is sandwiched between $\mathrm{Li}_{2} \mathrm{~N}$ layers. The iron concentrations, $x$, were measured by inductively coupled plasma mass spectrometry (ICP-MS), which enables a quantitative analysis on a parts per billion level (Fig. 1d). Structural parameters and selected $x$ values were determined by singlecrystal and powder X-ray diffraction and are in good agreement with earlier results ${ }^{22,27-29}$. To support our findings we present details of crystal growth procedure and chemical analysis (see Methods), as well as X-ray powder diffraction (Supplementary Fig. 2; Supplementary Note 2) and X-ray single-crystal diffraction (Supplementary Tables 1 and 2; Supplementary Note 3).

The samples are air sensitive in powder form but visual inspection and magnetic measurements revealed no significant decay of larger single crystals on a timescale of hours. As stated in Gregory $^{30}$, this is probably 'due, somewhat perversely, to the formation of a surface film of predominantly LiOH'. Covering the samples with a thin layer of Apiezon $\mathrm{M}$ grease further protects the sample and no degradation was observed over a period of several weeks. Single crystals that had been exposed to air for a few minutes and were stored afterwards in an inert atmosphere (argon or nitrogen) did not change their magnetic properties on a timescale of 3 months. The electrical resistivity at room temperature is estimated to be $\rho>10^{5} \Omega \mathrm{cm}$ for all studied $x$.

Magnetization. Extreme magnetic anisotropy of $\mathrm{Li}_{2}\left(\mathrm{Li}_{1-x} \mathrm{Fe}_{x}\right) \mathrm{N}$ is conspicuously evident in the magnetization measurements shown in Fig. 2a,b for two very different Fe concentrations of $x=0.0032$ and $x=0.28$. For magnetic field applied along the $\mathrm{c}$ axis, $H \| \mathrm{c}$, the magnetization is constant for $\mu_{0} H>1 \mathrm{~T}$ (starting from the fieldcooled state) with a large saturated moment of $\mu_{\text {sat }}^{\| \mathrm{c}} \approx 5 \mu_{\mathrm{B}}$ per Fe atom. In contrast, the magnetization for $H \perp c$ is smaller and slowly increases with field. A linear extrapolation to higher magnetic fields yields huge anisotropy fields of $\mu_{0} H_{\text {ani }} \approx 88 \mathrm{~T}$ $(x=0.0032)$ and $\mu_{0} H_{\mathrm{ani}} \approx 220 \mathrm{~T}(x=0.28)$ defined as the field strength where both magnetization curves intersect. The larger magnetic anisotropy field for $x=0.28$ is reflected in the larger coercivity field of $\mu_{0} H_{c}=11.6 \mathrm{~T}$ found for this concentration. The inverse magnetic susceptibility, $\chi^{-1}=H / M$, roughly follows a Curie-Weiss behaviour for $\mathrm{T} \gtrsim 150 \mathrm{~K}$ and is strongly anisotropic 
a

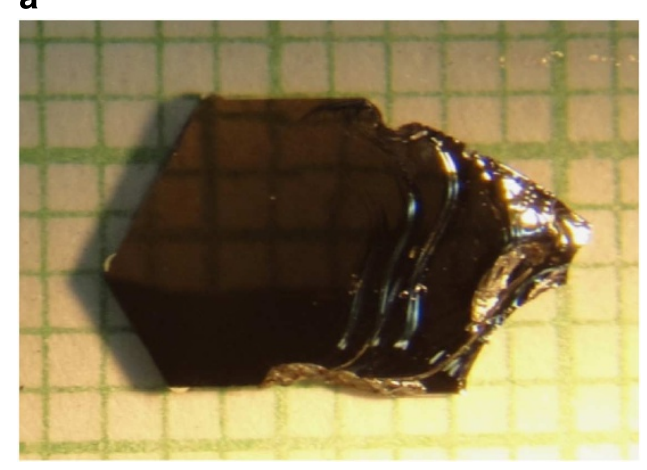

b

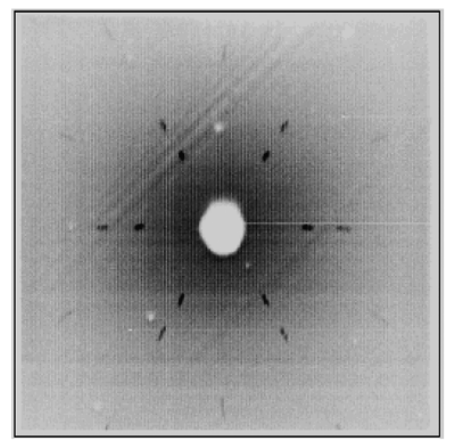

C

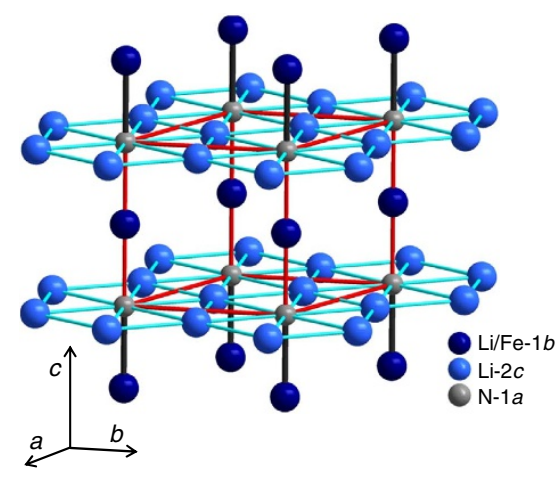

d

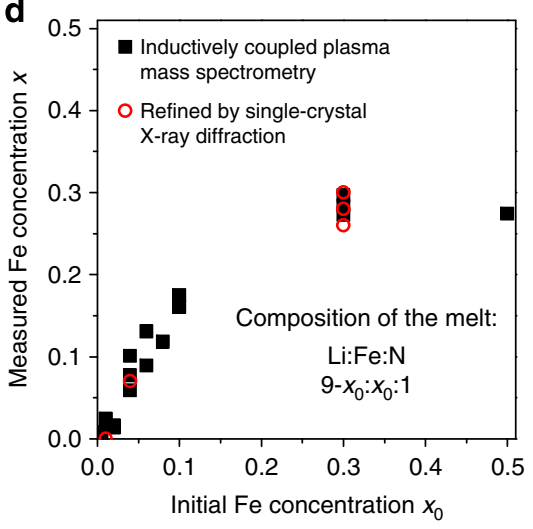

Figure 1 | Basic structural properties. (a) Single crystal of $\mathrm{Li}_{2}\left(\mathrm{Li}_{0.90} \mathrm{Fe}_{0.10}\right) \mathrm{N}$ on a millimetre grid and (b) corresponding Laue-back-reflection pattern. The crystal is not transparent and the faint grid pattern is a reflection off of the lens and the flat surface of the reflecting top facet. (c) Crystal structure with $\mathrm{Li}_{2} \mathrm{~N}$ layers separated by a second Li site, which is partially occupied by Fe. The unit cell of the hexagonal lattice is indicated by red lines. (d) Measured Fe concentration $x$ as a function of the Fe concentration in the melt $x_{0}$. For small concentrations, $x$ tends to be larger than $x_{0}$, however, a plateau in $x$ as a function of $x_{0}$ emerges for $x_{0} \gtrsim 0.3$. $x$ was determined by both ICP-MS (see Methods) and refinement of single-crystal X-ray diffraction data (see Supplementary Fig. 1).

over the whole investigated temperature range (Fig. 2c). The corresponding Weiss temperatures are $\Theta_{\mathrm{W}}^{\perp c}=-80(10) \mathrm{K}$ and $\Theta_{\mathrm{W}}^{\| \mathrm{c}}=100(10) \mathrm{K}$. In the following we discuss only measurements with $H \| \mathrm{c}$ since available laboratory fields do not allow saturation of the magnetization for $H \perp \mathrm{c}$. Both $\mu_{\text {sat }}^{\| \mathrm{c}}$ and $\mu_{\text {eff }}^{\| \mathrm{c}}$ were found to be largely independent of the Fe concentration but for a small tendency to decrease with increasing $x$ (Fig. 1d, see Supplementary Note 4 for error analysis). The average values $\mu_{\mathrm{sat}}^{\| \mathrm{c}}=4.8(4) \mu_{\mathrm{B}}$ and $\mu_{\text {eff }}^{\| \mathrm{c}}=6.5(4) \mu_{\mathrm{B}}$ are in good agreement with theoretical calculations for $x=0.17$ (ref. 24). Furthermore, the obtained effective moment is surprisingly close to the $\mu_{\text {eff }}=6.6 \mu_{\mathrm{B}}$ expectation value of a fully spin-orbit coupled state (Hund's rule coupling), when assuming a $3 d^{7}$ configuration for the proposed $\mathrm{Fe}^{+1}$ state (for a discussion of this unusual valence see refs 22,24).

In the following, we focus on experimental results on the very dilute case that approaches an ideally non-interacting system of single magnetic atoms. The temperature dependence of the $M-H$ loops for $x=0.0032$ with a high density of data points are shown in Fig. 3a. The following three main observations can be made:

First, the magnetization curve is essentially temperature independent for $T<10 \mathrm{~K}$ with $\mu_{0} H_{\mathrm{c}}=3.4 \mathrm{~T}$, which indicates the irrelevance of thermal excitations. In contrast, $H_{\mathrm{c}}$ changes dramatically between 10 and $16 \mathrm{~K}$, indicating a distinct separation into a low- and a high-temperature behaviour.

Second, there are pronounced steps in the magnetization. An enlarged view on part of the $M-H$ loop, Fig. $3 \mathrm{~b}$, reveals steps in $M$ at $\mu_{0} H=0, \pm 0.15$ and $\pm 0.55 \mathrm{~T}$. Another, smaller but well defined, step occurs at $\mu_{0} H= \pm 5 \mathrm{~T}$. The step sizes are larger when $H$ approaches zero and decrease with increasing temperature in contrast to the step positions, which are independent of temperature. No steps could be resolved for $T \gtrsim 16 \mathrm{~K}$. The steps are strongly suppressed with increasing $x$ (Supplementary Fig. 3), most likely caused by an increasing $\mathrm{Fe}-\mathrm{Fe}$ interaction and vanish for $x=0.28$ (Fig. 2b).

Third, the $M-H$ loop at $T=2 \mathrm{~K}$ starts from a saturated state where the Fe magnetic moments appear to be aligned parallel to the field, $\mu \| H$ with $H \|$ c. The first step occurs already in the first quadrant (upper right corner) when $H$ approaches zero from positive values. Similar behaviour was observed in lanthanidebased mononuclear SMMs ${ }^{15}$, whereas SMMs based on clusters show no corresponding steps in the first quadrant ${ }^{1}$. A decrease of the magnetization in the first quadrant, where $H$ is still parallel to the initial magnetization, is incompatible with the $c$ axis being the easy axis. Rather, the moment seems to be tilted away from the $c$ axis and the reduced magnetization is the projection of the moment along the $c$ axis (see Discussion). Notice that an axis canted away from the $c$ axis would be 12 - or 24 -fold degenerate (depending on whether it is oriented along a high-symmetry direction like $h 0 l$ or not). Accordingly, the magnetization perpendicular to the $\mathrm{c}$ axis $(H \perp \mathrm{c}, M \perp \mathrm{c})$ is still small in zero field because the perpendicular components of the tilted moments cancel out.

In contrast to a common ferromagnet, the $M-H$ loops not only depend on temperature but also show a pronounced, characteristic dependence on the sweep rate of the applied magnetic field. Figure $3 \mathrm{c}, \mathrm{d}$ shows $M-H$ loops at $T=2 \mathrm{~K}$ for sweep rates between 15 and $1.1 \mathrm{mT} \mathrm{s}^{-1}$, corresponding to a total time between $40 \mathrm{~min}$, 
a

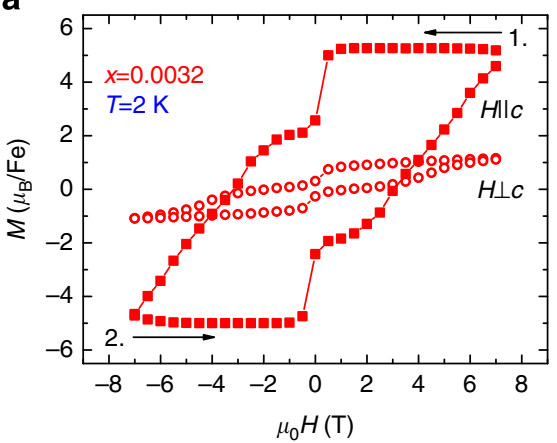

C

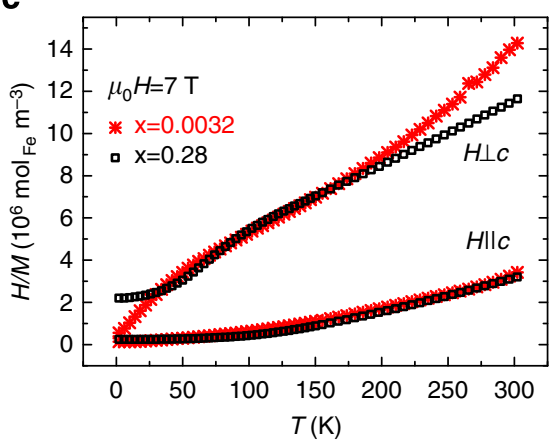

b

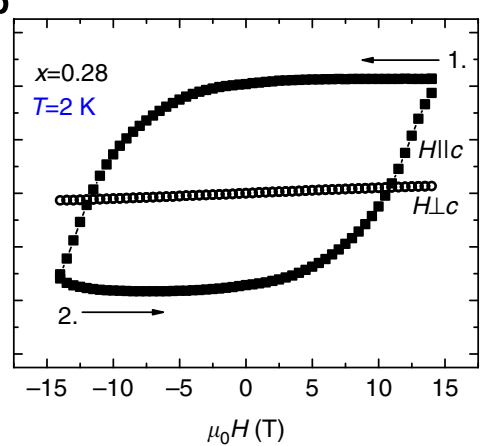

d

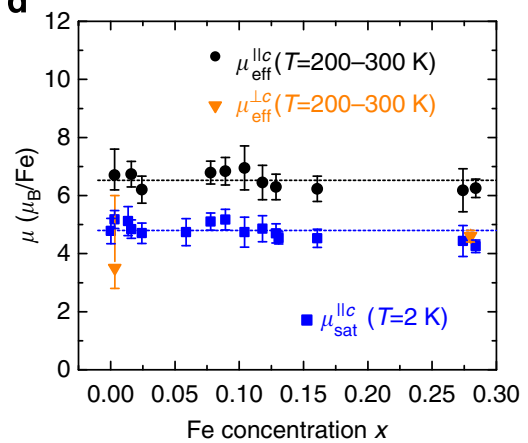

Figure 2 | Basic magnetic properties of $\mathbf{L i}_{\mathbf{2}}\left(\mathbf{L i}_{\mathbf{1}}{ }_{\mathbf{x}} \mathbf{F e}_{\mathbf{x}}\right) \mathbf{N}$. $(\mathbf{a}, \mathbf{b})$ Large hysteresis in the magnetization, $M(H)$, and a pronounced anisotropy depending on the orientation of the applied magnetic field, $H$, with respect to the crystallographic axes for $x=0.0032$ and $x=0.28$. Whereas $M$ can be saturated for $H \| c$, it is slowly increasing with $\mathrm{H}$ for $\mathrm{H} \perp \mathrm{c}$ up to the highest available fields. (c) The temperature dependence of the inverse magnetization for $x=0.28$ follows a Curie-Weiss law for $T \gtrsim 150 \mathrm{~K}$ with effective moments of $\mu_{\mathrm{eff}}^{\perp c}=4.6(3) \mu_{\mathrm{B}}$ for $H \perp c$ and $\mu_{\mathrm{eff}}^{\| c}=6.3(4) \mu_{\mathrm{B}}$ for $H \| c$. Similar behaviour is observed for the two orders of magnitude lower concentration of $x=0.0032$, where the deviation for $H \perp c$ at $T>200 \mathrm{~K}$ can be caused by a temperature-independent diamagnetic background of the $\mathrm{Li}_{3} \mathrm{~N}$ host, which is negligible for higher Fe concentrations. (d) Both $\mu_{\text {sat }}$ and $\mu_{\text {eff }}$ were found to be largely independent of $x$ over the whole investigated range of $x=0.00028-0.28$. The error bars are calculated based on the errors in assessing the sample mass and the Fe concentration and, for $x=0.0032$, on a diamagnetic contribution of the $\mathrm{Li}_{3} \mathrm{~N}$ host. The average values are $\mu_{\mathrm{sat}}^{\| c}=4.8(4)$ and $\mu_{\mathrm{eff}}^{\| c}=6.5(4) \mu_{\mathrm{B}}$.

and $9 \mathrm{~h}$ and $10 \mathrm{~min}$, respectively, for the whole loop. The step sizes at $\mu_{0} H= \pm 0.15$ and $\pm 0.55 \mathrm{~T}$ hardly depend on the sweep rate, whereas the step size at $H=0$ does. A low sweep rate dependence of the step size is in accordance with a large tunnelling gap, which is much smaller than the energy gap ${ }^{31}$ (for a convenient description of the relation between sweep rate and tunnelling gap see, for example, Wernsdorfer and Sessoli ${ }^{32}$ and references therein). Although the field values for the steps do not change significantly with sweep rate, the width of the whole hysteresis loop (and therefore $H_{\mathrm{c}}$ ) does (Fig. 3c). Pronounced sweep rate dependence of $H_{c}$ is observed also for higher $\mathrm{Fe}$ concentrations-see Supplementary Fig. 3. The observation of smaller $H_{c}$ values in slower measurements clearly reveals the dynamic nature of the hysteresis. This observation motivated a detailed study of the time dependence of the magnetization, which is presented in the following section.

Relaxation. When a magnetic moment is subject to a change in the applied magnetic field, it will take a finite relaxation time to reach the equilibrium state (see, for example, Cullity ${ }^{33}$ ). The sweep rate dependence of the hysteresis loops indicates a timescale of several hours for the relaxation in $\mathrm{Li}_{2}\left(\mathrm{Li}_{1-x} \mathrm{Fe}_{x}\right) \mathrm{N}$. This is so slow that the change of the magnetization with time, $M(t)$, during the relaxation process can be directly measured by standard laboratory magnetometers. Three procedures are schematically shown in the left panels of Fig. 4 (see Supplementary Note 4 for detailed measurement protocols). The corresponding experimental data are in the panels to the right.
Figure $4 \mathrm{a}-\mathrm{c}$ shows the relaxation after switching-on the field. $M(t)$ increases rapidly for $T=16 \mathrm{~K}$ and the magnetization is constant after $t \sim 500 \mathrm{~s}$. The relaxation is becoming markedly slower as the measurement temperature is reduced to $T=10 \mathrm{~K}$, and $M(t)$ keeps increasing up to the maximum measurement time. However, below $10 \mathrm{~K}, M(t)$ is only weakly temperature dependent in $\mu_{0}=1 \mathrm{~T}$ and essentially temperature independent in $\mu_{0} H=2 \mathrm{~T}$. This temperature independence is inconsistent with a thermally activated relaxation process. The inset of Fig. $4 \mathrm{c}$ shows an enlarged region of the plot. No correlation of relaxation with temperature is apparent. The relaxation for $T>16 \mathrm{~K}$ is too fast to be measured directly and $M(t)$ becomes immediately constant once the field is stabilized. The relaxation process shows clear anomalies at the resonance fields (step positions in $M-H$ loops) as shown in Supplementary Fig. 4.

Figure $4 \mathrm{~d}-\mathrm{f}$ shows the relaxation after switching-off the field. The relaxation is fast for $T=16 \mathrm{~K}$ but suppressed for lower temperatures. However, the decrease is non-uniform with temperature and markedly smaller at the lowest temperatures. $M(t)$ can be fitted to a stretched exponential

$$
M(t)=M_{0} \exp \left[-(t / \tau)^{\beta}\right]
$$

with $M_{0}=M(t=0)$ and $\tau$ is the relaxation time (for details regarding the exponent $\beta$ see Supplementary Fig. 5 and Supplementary Note 4). For $T>16 \mathrm{~K}, \tau$ is too small to be measured directly but can be determined from the out-of-phase part of the alternating current magnetic susceptibility, $\chi^{\prime \prime}(T)$. The inset in Fig. $4 \mathrm{f}$ shows maxima in $\chi^{\prime \prime}(T)$ at temperatures $T_{\max }$, 
a

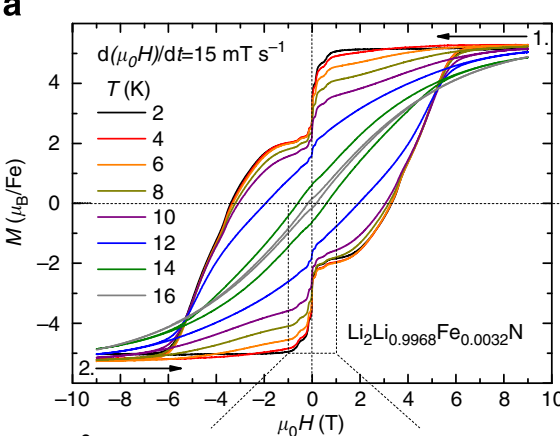

b

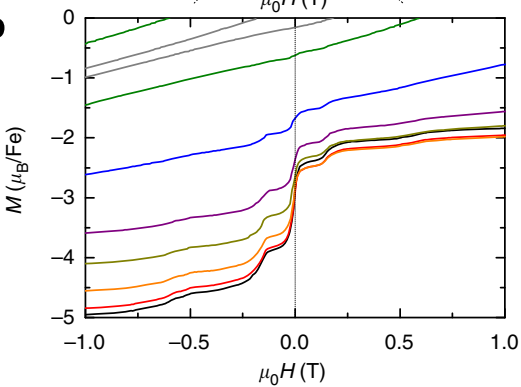

C
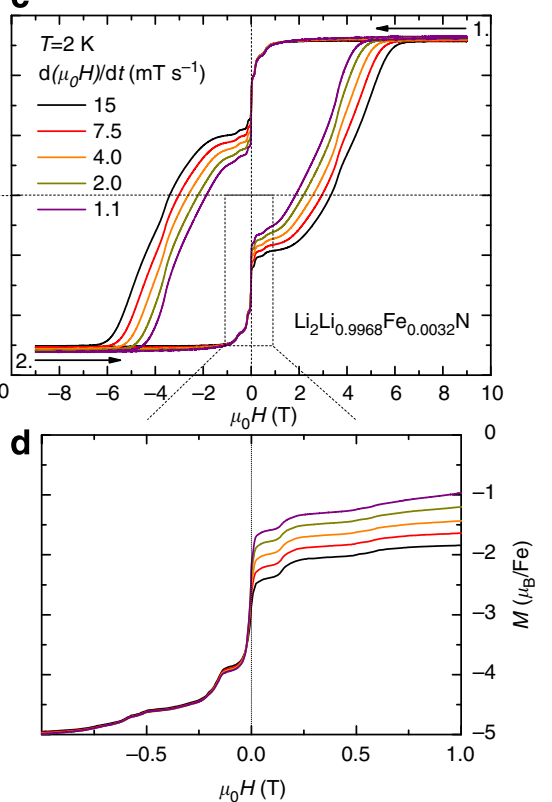

Figure 3 | Temperature and sweep rate dependence of the magnetization of $\mathbf{L i}_{\mathbf{2}}\left(\mathbf{L i}_{\mathbf{1}}{ }_{\mathbf{x}} \mathbf{F e}_{\mathbf{x}}\right) \mathbf{N}$ for $\mathbf{x}=\mathbf{0 . 0 0 3 2}, \mathbf{H} \| \mathbf{c}$. (a) Hysteresis emerges for $T \leq 16 \mathrm{~K}$ and the coercivity fields, $H_{\mathrm{c}}$, are essentially temperature independent below $T=10 \mathrm{~K}$. (b) The clear steps in the magnetization are smeared out with increasing temperature and disappear for $T \gtrsim 16 \mathrm{~K}$. (c) The pronounced sweep rate dependence of the magnetization reveals the dynamic nature of the hysteresis. (d) Only the step at $H=0$ depends significantly on the sweep rate, indicating that the relaxation at the smaller steps at $\mu_{0} H= \pm 0.15$ and $\pm 0.55 \mathrm{~T}$ is fast on the timescale of the experiment. The step at $H=0$ is attributed to a flip of the Fe magnetic moment from below to above the crystallographic $a-b$ plane. The energy barrier associated with this transition seems to dominate the relaxation process and forms the global maximum in the magnetic anisotropy energy. The smaller steps are accordingly associated with tunnelling through smaller, local maxima.

which increase with the excitation frequency $f$. The maximum in $\chi^{\prime \prime}(T)$ corresponds to a maximum in the energy absorption of the alternating magnetic field by the sample and shows that the relaxation time equals the timescale of the experiment, that is, $\tau=1 / f$ at $T=T_{\max }$. The values obtained for $\tau$ are shown in Fig. $4 \mathrm{f}$ in the form of an Arrhenius plot. For $T \geq 16 \mathrm{~K}, \tau$ follows a linear (Arrhenius) behaviour with good agreement between directly and indirectly measured values. A fit of $\tau$ to a thermally activated law $\left(\tau=\tau_{0} \exp \left[\Delta E / k_{\mathrm{B}} T\right]\right.$, dashed line) gives an energy barrier of $\Delta E / k_{\mathrm{B}}=430 \mathrm{~K}$ and a pre-exponential factor $\tau_{0}=2.8 \times 10^{-10} \mathrm{~s}$. For $T<16 \mathrm{~K}, \tau$ deviates significantly from Arrhenius behaviour and decreases much slower with decreasing temperature than expected for a thermally activated relaxation. A plateau, as shown in Fig. $4 \mathrm{f}$, has been found in $\mathrm{SMMs}^{2,34}$ and is a clear fingerprint of quantum tunnelling.

Figure $4 \mathrm{~g}-\mathrm{i}$ shows the relaxation in negative fields. This method is a variation of the previous one where the field is not simply switched-off but ramped to a negative value opposite to the initial direction. As shown by the large magnetic hysteresis and the relaxation measurements above, the dominating energy scale of the system lies in the region of several Tesla acting on a few $\mu_{\mathrm{B}}$. Therefore, a magnetic field of a few milliTesla can, at most, merely change the energy-level scheme. Furthermore, applying a negative field is expected to accelerate the decrease of $M(t)$. In contrast to both assumptions, small fields of $\mu_{0} H=-1$ to $-10 \mathrm{mT}$ have a dramatic effect on the relaxation time and lead to an increase of $\tau$ by several orders of magnitude (black curves in Fig. 4h). In fact, the relaxation in $\mu_{0} H=-10 \mathrm{mT}$ is becoming so slow that an accurate determination of $\tau$ is not possible within $4,000 \mathrm{~s}$ but requires much longer measurement times (which is beyond the scope of this publication). The main effect of the small negative field is likely a destruction of the tunnelling condition by lifting the zero-field degeneracy-resonant tunnelling of the magnetization occurs only between degenerate states ${ }^{1}$.
In intermediate fields of $\mu_{0} H=-50$ to $-500 \mathrm{mT}$, the magnetization decreases rapidly until the applied field is stable (that is, after $50 \mathrm{~s}$ for $\mu_{0} H=-500 \mathrm{mT}$ ), followed by a significantly slower relaxation. This indicates the presence of two timescales for the relaxation, a fast one and a slow one. The slow relaxation can be associated with overcoming the large energy barrier in the $a-b$ plane. However, the flipped moments are still not (anti-)parallel to the $c$ axis (Fig. 5a). The fast relaxation can be associated with the subsequent, full alignment of the magnetic moments along the $\mathrm{c}$ axis by overcoming smaller, local maxima in the magnetic anisotropy energy (corresponding to the smaller steps in the $M-H$ loop, see Fig. 5b). Only larger fields of $\left|\mu_{0} H\right|>1 \mathrm{~T}$ cause a faster decrease of $M(t)$ when compared with zero field (Fig. 4i). A fast relaxation on the timescale of the experiment is reached at $\mu_{0} H=-7 \mathrm{~T}$ where $M(t)$ is saturated after $t \approx 2,000 \mathrm{~s}$. Accordingly, the magnetization reaches saturation in a similar short time after applying a field of $\mu_{0} H=7 \mathrm{~T}$ to an unmagnetized sample (analogue to Fig. $4 a-c$, not shown). It should be noted that the time dependencies, as presented in this section, are not restricted to low $\mathrm{Fe}$ concentrations. Clear relaxation effects are observed through the whole concentration range with the tendency to slow down with increasing $x$.

\section{Discussion}

Our work shows that $\mathrm{Li}_{2}\left(\mathrm{Li}_{1-x} \mathrm{Fe}_{x}\right) \mathrm{N}$ not only has a clear and remarkable anisotropy, generally not associated with $\mathrm{Fe}$ moments, but also shows time dependence more consistent with SMM systems. The strong correlation of relaxation time and coercivity field (Fig. 4f) indicates that the slow relaxation leads to magnetic hysteresis for $x \ll 1$, that is, the dilute system is not a ferromagnet and hysteresis emerges from a slow decay of a polarized, paramagnetic state. Whether this holds true for the dense system $(x=0.28)$, too, is not settled at this point-below 
a

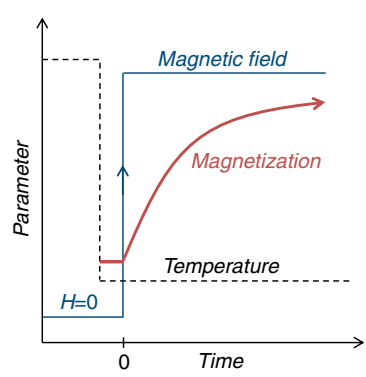

d

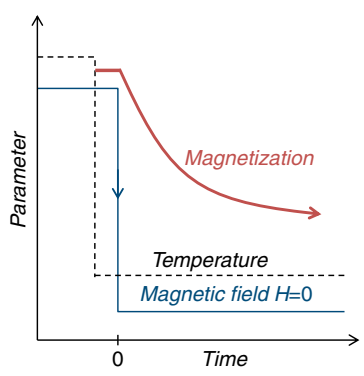

g

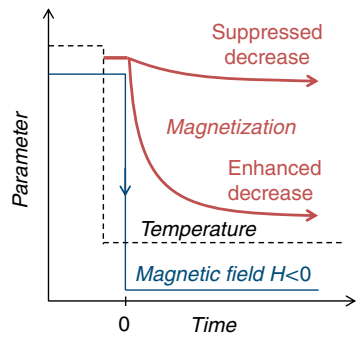

b

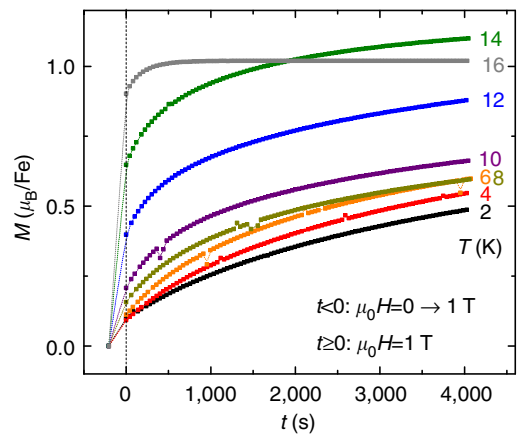

e

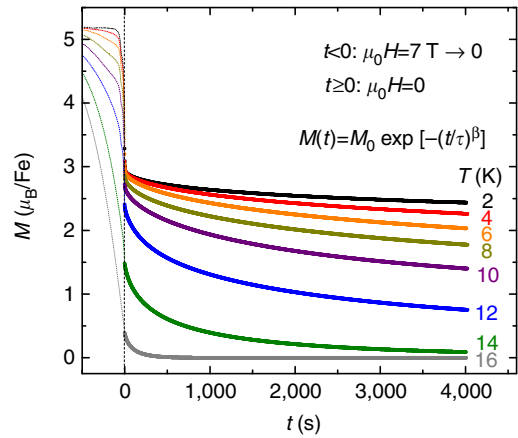

h

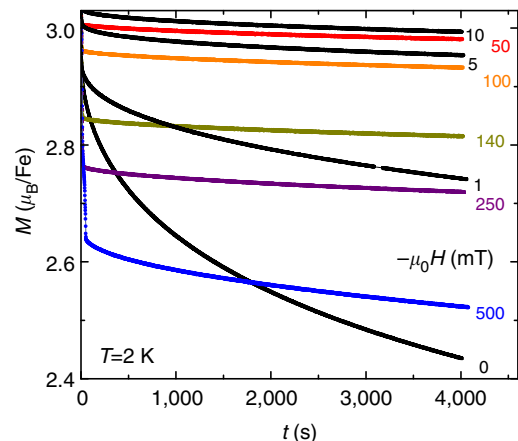

c

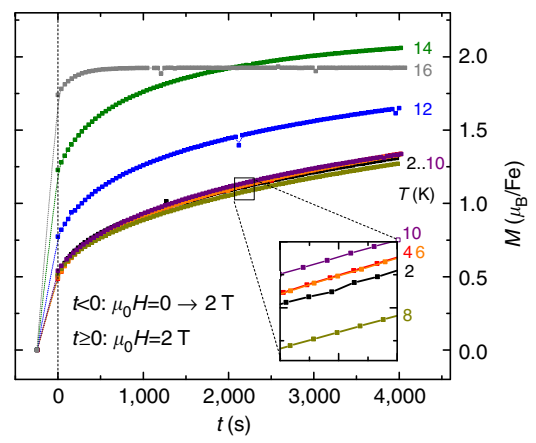

f

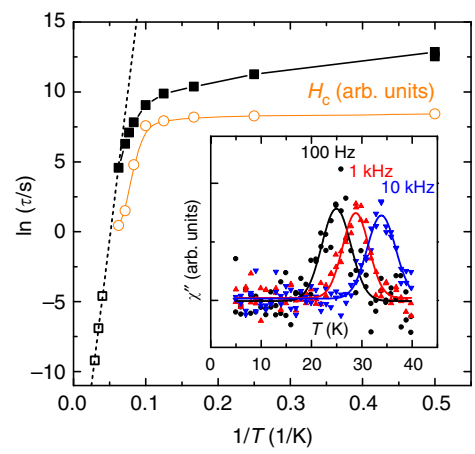

i

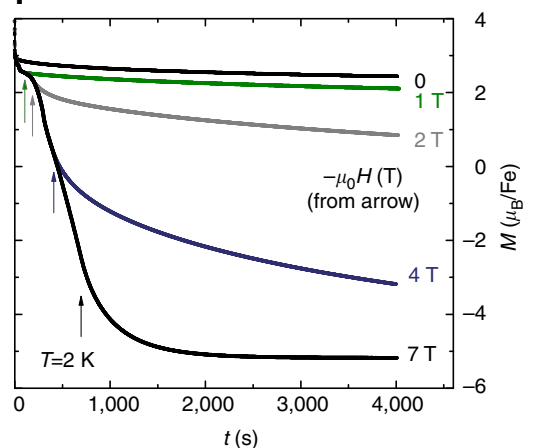

Figure 4 | Time dependence of the magnetization. $(\mathbf{a}, \mathbf{d}, \mathbf{g})$ Schematics of different relaxation processes and corresponding experimental data on $\mathrm{Li}_{2}\left(\mathrm{Li}_{0.9968} \mathrm{Fe}_{0.0032}\right) \mathrm{N}$ to the right. (b) The increase in the magnetization as a function of time, $M(t)$, below $T=10 \mathrm{~K}$ is only weakly temperature dependent for $\mu_{0} H=1 \mathrm{~T}$. (c) Increasing the applied field to $\mu_{0} \mathrm{H}=2 \mathrm{~T}$ leads to an essentially temperature-independent relaxation below $T=10 \mathrm{~K}$, which is inconsistent with a thermally activated relaxation process. (e) Decrease in $M(t)$ after ramping the field from $\mu_{0} H=7 T$ to $0 . M(t)$ is fit to a stretched exponential function and the obtained relaxation times, $\tau$, are shown as closed squares in the form of an Arrhenius plot in (f). (f) $\tau$ determined from the imaginary part of the alternating current magnetic susceptibility, $\chi^{\prime \prime}(T)$ (inset), is shown by open squares. Thermally activated behaviour is observed at higher temperatures (dashed line). The formation of a plateau towards lower temperatures is incompatible with thermally activated behaviour and indicates the relevance of quantum tunnelling. The coercivity field (open circles, sweep rate $15 \mathrm{mTs}^{-1}$ ) shows similar temperature dependence, indicating slow relaxation as the origin of spontaneous magnetization. (g) Small negative fields of a few milliTesla, applied opposite to the initial applied field of $\mu_{0} H=7 \mathrm{~T}$, significantly reduce the decrease in $M(t)$ compared with $H=0$. (i) In contrast, $M(t)$ decreases faster in larger negative fields of $\left|\mu_{0} H\right| \geq 1 \mathrm{~T}$.

$T \approx 65 \mathrm{~K}$ the ordering appears to be static on the timescale of Mössbauer spectroscopy 22,23 (performed on polycrystalline material, $x=0.16$ and 0.21 ).

A possible schematic for the magnetic moment orientation and the magnetic anisotropy energy is depicted in Fig. 5. This model is purely based on the observed plateaus in the $M-H$ measurements and, beyond the extreme uniaxial symmetry of the iron environment, the origin of the magnetic anisotropy of $\mathrm{Li}_{2}\left(\mathrm{Li}_{1-}\right.$ $\left.{ }_{x} \mathrm{Fe}_{x}\right) \mathrm{N}$ is still unclear. The anisotropy found in SMMs is based on the magnetic interactions within the transition metal cluster or in the single-ion anisotropy of lanthanide ions (or on both). For the latter case, the anisotropy is caused by the crystal electric field that acts as perturbation on the multiplet ground state of the lanthanide ion, which is determined by Hund's rule coupling. Neither of these conceptionally simple scenarios is applicable for $\mathrm{Li}_{2}\left(\mathrm{Li}_{1-x} \mathrm{Fe}_{x}\right) \mathrm{N}$ since the $\mathrm{Fe}$ atoms are too dilute to strongly interact with each other for $x \ll 1$ and the crystal electric field is too strong to be regarded as a small perturbation when compared with the spin-orbit coupling. Instead we are left with the complex determination of the energy-level scheme of the relevant $\mathrm{Fe}-3 d$ electrons. Calculations based on LDA predict a counter-intuitive energy-level scheme with the $3 d_{\mathrm{z}}^{2}$ level having the lowest energy followed by partially occupied $d_{x^{2}-y^{2}}, d_{x y}$ levels ${ }^{24}$. A very similar energy-level scheme was recently found for a mononuclear Febased SMM $^{19}$ sharing a similar structural motif as the Fe site in $\mathrm{Li}_{2}\left(\mathrm{Li}_{1-x} \mathrm{Fe}_{x}\right) \mathrm{N}$ : a linear $\mathrm{Fe}^{+1}$ complex, which seems to be an 
a

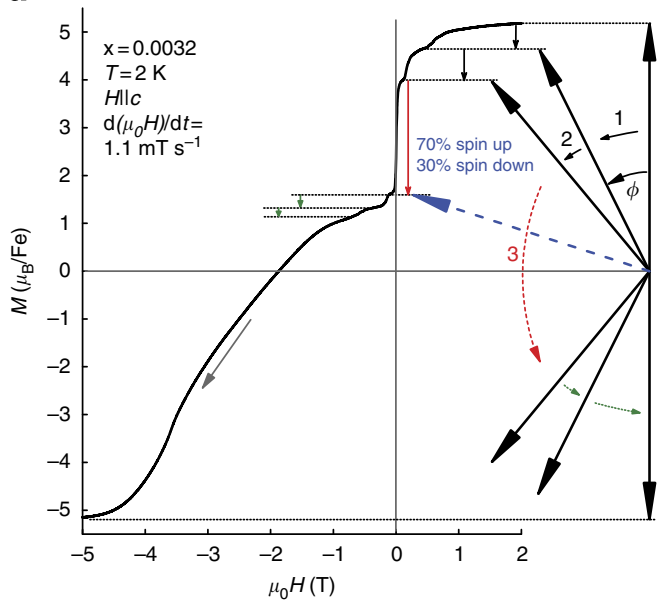

b

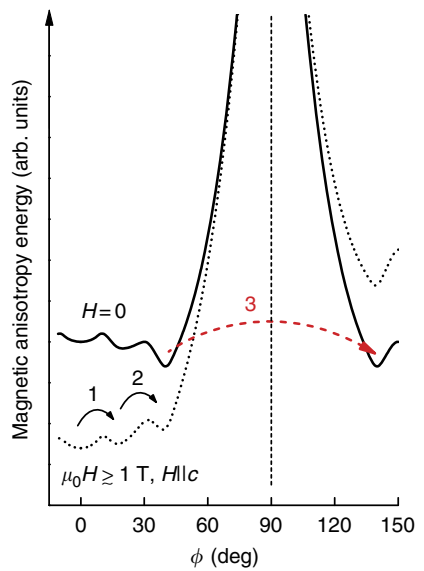

Figure 5 | Magnetic moment orientation and anisotropy energy. (a) Magnetization of $\mathrm{Li}_{2}\left(\mathrm{Li}_{1}-{ }_{x} \mathrm{Fe}_{x}\right) \mathrm{N}$ and a corresponding possible orientation of the magnetic moment. This simplified model assumes a constant magnitude of the magnetic moment independent of the orientation with respect to the c axis ( $\phi$ is the angle between moment and c axis). The plateau after step 2 corresponds to $\phi \sim 40^{\circ}$. The large jump in $M(H)$ at $H \sim 0$ is in accordance with a reorientation of $30 \%$ of the Fe magnetic moments from spin-up $\left(\phi \sim 40^{\circ}\right)$ to spin-down $\left(\phi \sim 140^{\circ}\right)$. A further rapid reorientation for $H<0$ is blocked because the resonance condition for magnetic tunnelling is destroyed for $H \neq 0$. (b) Possible schematic of the magnetic anisotropy energy as a function of the angle to the crystallographic $\mathrm{c}$ axis. The global minimum in zero field appears at an angle of $\phi \sim 40^{\circ}$. Adding a sufficiently large Zeeman term for an applied field along the $\mathrm{c}$ axis (dotted line) leads to a global minimum located at $\phi=0$. The large step in the $M-H$ loop occurring at zero field is associated with the resonant tunnelling through the large energy barrier centred at $\phi=90^{\circ}$. Accordingly, the smaller steps correspond to transitions from a local to the (actual) global minimum in the total magnetic energy.

essential ingredient for the emergence of unquenched orbital moments and large magnetic anisotropy.

Even though the exact microscopic origin of the energy barriers separating the magnetic states in $\mathrm{Li}_{2}\left(\mathrm{Li}_{1-x} \mathrm{Fe}_{x}\right) \mathrm{N}$ is not yet understood, we argue that quantum tunnelling has to be invoked to overcome them. From the Arrhenius fit we find $\Delta / k_{\mathrm{B}}=430 \mathrm{~K}$ (Fig. 4f). Magnetization measurements on single crystals allow for a second method to estimate the barrier height: when $H$ reaches the anisotropy field, all moments are aligned along the field and the energy barrier is overcome. This gives rise to $\Delta E / k_{\mathrm{B}} \approx \mu_{0} H_{\text {ani }} \times \mu_{\text {sat }} / k_{\mathrm{B}} \approx 88 \mathrm{~T} \times 5 \mu_{\mathrm{B}} / k_{\mathrm{B}}=300 \mathrm{~K}$ in reasonable agreement with the above result. This value also agrees with the LDA-based calculation of the anisotropy energy for $x=0.17$ ( $\Delta /$ $\left.k_{\mathrm{B}}=278 \mathrm{~K}\right)^{24}$. Such a large barrier cannot be overcome thermally at $T=2 \mathrm{~K}$-an estimation of the relaxation time from thermally activated law and $\Delta / k_{\mathrm{B}}=278 \mathrm{~K}$ yields $\tau>10^{43}$ years (give or take a millennium). Therefore, a tunnelling process is likely involved in the relaxation process where the applied magnetic field shifts the energy levels in and out of degeneracy in full analogy to SMMs. At this point it is not clear which levels of the single ion are brought in resonance by the applied magnetic field to explain the smaller steps in the $M-H$ loops. Hyperfine interactions, as invoked for lanthanide-based $\mathrm{SMMs}^{15}$ and $\mathrm{LiY}_{0.998} \mathrm{Ho}_{0.002} \mathrm{~F}_{4}$ (ref. 35), are probably too weak to account for steps at applied fields of $\mu_{0} H=0.5 \mathrm{~T}$ in particular since $98 \%$ of the $\mathrm{Fe}$ atoms do not carry a nuclear moment.

Energy barriers of similar $\left(\Delta / k_{\mathrm{B}}=300 \mathrm{~K}^{19,36}\right)$ or even higher size $\left(\Delta / k_{\mathrm{B}}=800 \mathrm{~K}^{37}\right)$ have been observed in mononuclear SMMs. However, the remanent magnetization for these samples is very small caused by short relaxation times at low temperatures, that is, $\tau$ deviates from Arrhenius behaviour with a relaxation time at the plateau in the order of seconds-in contrast to $\tau \sim 10^{5} \mathrm{~s}$ found for $\mathrm{Li}_{2}\left(\mathrm{Li}_{1-x} \mathrm{Fe}_{x}\right) \mathrm{N}$. The fortunate combination of a deviation from Arrhenius behaviour at high temperatures $(T>10 \mathrm{~K})$ with large values at the plateau leads to the extreme coercivities presented here. For potential data storage applications and stable magnetic materials, it is desirable to increase $\tau$ by suppressing the tunnel effect, which can be achieved by enhanced exchange coupling ${ }^{11,38}$. On the other hand, studying tunnelling phenomena by itself requires isolated magnetic moments. Both goals can be satisfied by varying the $\mathrm{Fe}$ concentration in $\mathrm{Li}_{2}\left(\mathrm{Li}_{1-x} \mathrm{Fe}_{x}\right) \mathrm{N}$ accordingly. Thus for low $\mathrm{Fe}$ concentration of $x=0.0032$, statistically, $98 \%$ of the $\mathrm{Fe}$ atoms have only $\mathrm{Li}$ as the nearest neighbours in the $a-b$ plane and $\mathrm{Fe}-\mathrm{Fe}$ interactions are negligible. Larger $\mathrm{Fe}$ concentrations show higher coercivity fields at the lowest temperatures (Supplementary Fig. 3), which are caused by larger relaxation times, indicating that $\mathrm{Fe}-\mathrm{Fe}$ interactions are indeed detrimental to tunnelling. Provided the energy barriers can be further enhanced and tunnelling appropriately controlled, this opens a route for the creation of hard permanent magnets from cheap and abundant elements.

One remaining question is: why is quantum tunnelling so elusive in inorganic compounds? Besides our discovery in $\mathrm{Li}_{2}\left(\mathrm{Li}_{1-x} \mathrm{Fe}_{x}\right) \mathrm{N}$, we are aware of only one other family of inorganic compounds showing macroscopic quantum tunnelling effects of the magnetization: $\mathrm{LiY}_{0.998} \mathrm{Ho}_{0.002} \mathrm{~F}_{4}$ (refs 26,35) and related systems. However, the characteristic energy scales are two orders of magnitude smaller than in $\mathrm{Li}_{2}\left(\mathrm{Li}_{1-x} \mathrm{Fe}_{x}\right) \mathrm{N}$ and magnetic hysteresis emerges only below $T=200 \mathrm{mK}$ with coercivity fields of $\mu_{0} H_{c}=30 \mathrm{mT}$. To observe a macroscopic quantum effect such as tunnelling of the magnetization, the interaction between the magnetic moments has to be small. In general, coupling leads to excitations (modes), which lead to dissipation that destroys the quantum state. This rules out systems with dense, interacting moments. Diluted systems of local magnetic moments have been the subject of extensive research mainly to study the Kondo effect. This necessarily requires metallic samples; the magnetic moments are not isolated but coupled to the electron bath, which again leads to dissipation and the destruction of the quantum state. Insulating samples with diluted or non-interacting magnetic moments have been far less studied-diluted magnetic semiconductors are explicitly excluded from this statement because of their finite carrier density. A possible explanation for the absence of magnetic tunnelling 
in insulators is a Jahn-Teller distortion, which is frequently observed, for example, in lanthanide zircons of the form $\mathrm{RXO}_{4}$ (Kirschbaum $^{39}$ and references therein). According to the JahnTeller theorem ${ }^{40}$, the orbital degeneracy of Fe should cause a structural distortion also for $\mathrm{Li}_{2}\left(\mathrm{Li}_{1-}{ }_{x} \mathrm{Fe}_{x}\right) \mathrm{N}$ in order to reach a stable state. In the diluted case it will occur locally for Fe and not necessarily for the whole crystal. Lifting the orbital degeneracy corresponds to a zero-orbital angular momentum, which is the only fully non-degenerate state. Consequently, a Jahn-Teller distortion would lead to a loss of the magnetic anisotropy and a decay of the energy barrier. However, the Fe atom sits between two nitrogen neighbours, which provide the dominant bonding. These three atoms can be regarded as acting like a linear molecule, which is not subject to a Jahn-Teller distortion ${ }^{40}$.

To summarize, we demonstrated a huge magnetic anisotropy and coercivity in $\mathrm{Li}_{2}\left(\mathrm{Li}_{1-x} \mathrm{Fe}_{x}\right) \mathrm{N}$ and want to emphasize the three properties that are probably essential for the emergence of macroscopic quantum tunnelling: the compound is insulating, the orbital magnetic moment of $\mathrm{Fe}$ is not quenched and the $\mathrm{N}-\mathrm{Fe}-\mathrm{N}$ complex forms a linear molecule avoiding a Jahn-Teller distortion. These properties may serve as a basis for the design of materials featuring even higher characteristic energy scales.

\section{Methods}

Crystal growth. Starting materials were $\mathrm{Li}$ granules (Alfa Aesar, 99\%), $\mathrm{Li}_{3} \mathrm{~N}$ powder (Alfa Aesar, 99.4\%) and Fe granules (99.98\%). The mixtures had a molar ratio of Li:Fe: $\mathrm{N}=9-x_{0}: x_{0}: 1$ with $x_{0}=0-0.5$. A total mass of roughly $1.5 \mathrm{~g}$ was packed into a 3-cap Ta crucible ${ }^{41}$ inside an Ar-filled glovebox. The Ta crucible was sealed by arc melting under inert atmosphere of $\sim 0.6$ bar $\mathrm{Ar}$ and subsequently sealed in a silica ampoule. The $\mathrm{Li}-\mathrm{Fe}-\mathrm{N}$ mixture was heated from room temperature to $T=900^{\circ} \mathrm{C}$ over $4 \mathrm{~h}$, cooled to $T=750^{\circ} \mathrm{C}$ within $1.5 \mathrm{~h}$, slowly cooled to $T=500^{\circ} \mathrm{C}$ over $62 \mathrm{~h}$ and finally decanted to separate $\mathrm{Li}_{2}\left(\mathrm{Li}_{1-}{ }_{x} \mathrm{Fe}_{x}\right) \mathrm{N}$ crystals from the excess liquid. Single crystals of hexagonal and plate-like habit with masses $>100 \mathrm{mg}$ could be obtained. The maximum lateral sizes of $\approx 10 \mathrm{~mm}$ were limited by the crucible size where the crystal thickness is typically $\approx 1 \mathrm{~mm}$. Typically we found a few large single crystals with similar orientation clamped between the container walls above the bottom of the crucible and also several smaller ones attached to the bottom. We used pieces of the larger crystals for the magnetization measurements presented in this publication.

It should be noted that the smallest Fe concentration of $x=0.00028$, as measured by ICP-MS (see below), was obtained without intentional introduction of Fe in the melt. Fe was most likely introduced as an impurity from the starting materials or from the Ta crucible.

Chemical analysis with ICP-MS. The $\mathrm{Li}_{2}\left(\mathrm{Li}_{1-x} \mathrm{Fe}_{x}\right) \mathrm{N}$ samples were analysed using an inductively coupled plasma magnetic sector mass spectrometer (ICP-MS, Element 1, Thermo Scientific). The samples were introduced into the ICP via a low-flow nebulizer (PFA-100, Elemental Scientific Inc.) and double-pass spray chamber. The interface between the ICP and mass spectrometer was equipped with nickel sampler and skimmer (H-configuration) cones. The mass spectrometer was operated in medium resolution $(m / \Delta m=4,000)$ to separate the $\mathrm{Fe}^{+}$isotopes of interest from interfering species. The detector was operated in dual mode, allowing for the operating software to selectively switch between analogue and counting measurements. Prior to sample analysis, the torch position and instrumental operating parameters (Table 1) were adjusted for maximum peak height and signal stability. The main elements of interest for quantification were lithium (mass-tocharge ratio $m / z=7)$ and iron $(m / z=55.935$ and 56.935). Tantalum $(m / z=181)$ and calcium $(\mathrm{m} / z=43$ and 44$)$ were also measured to check for contamination from the crucible material and known impurities of the starting materials.

\section{Table 1 | ICP-MS operating parameters.}

\begin{tabular}{ll} 
RF power & $\mathbf{1 , 1 5 0 ~ \mathbf { ~ W }}$ \\
\hline Outer gas flow & $161 \mathrm{~min}^{-1}$ \\
Auxiliary gas flow & $1.5 \mathrm{Imin} \mathrm{min}^{-1}$ \\
Sample gas flow (argon) & $1.04 \mathrm{Imin}-1$ \\
Sampling position & $13 \mathrm{~mm} \mathrm{from} \mathrm{load} \mathrm{coil,} \mathrm{on} \mathrm{centre}_{\text {Signal ratios for tuning }} \mathrm{Ce}^{2+} / \mathrm{Ce}^{+} \approx 4 \%, \mathrm{CeO}^{+} / \mathrm{Ce}^{+} \approx 7 \%$ \\
and optimization & \\
\hline ICP-MS, inductively coupled plasma mass spectrometry; RF, radio frequency.
\end{tabular}

Following these initial analyses, a full isotopic spectrum $(\mathrm{m} / z=7-238)$ was measured in low resolution $(m / \Delta m=300)$ for several representative samples to check for any other possible sources of contamination. Carbon, nitrogen and oxygen could not be measured due to high background levels contributed by the acid solution, the argon gas and the instrumental components. Minor amounts of tantalum and calcium were measured in the Li-Fe-N samples. All samples contained less than 0.4 mass $\%$ calcium (corresponding to $\mathrm{Li}_{1-\delta} \mathrm{Ca}_{\delta}$ with $\delta<0.007$ ) and less than 0.1 mass $\%$ tantalum (corresponding to $\mathrm{Li}_{1-\delta} \mathrm{Ta}_{\delta}$ with $\delta<0.0004$ ). The combined concentrations of all other contaminant elements comprised far less than 0.01 mass $\%$ of the solid samples.

All of the $\mathrm{Li}_{2}\left(\mathrm{Li}_{1-x} \mathrm{Fe}_{x}\right) \mathrm{N}$ samples were dissolved for ICP-MS analysis. Approximately 5-25 mg of solid sample was weighed accurately into an acid vapour-washed Teflon bottle on a balance. A small amount $(2-4 \mathrm{~g})$ of cold $\left(\sim 3^{\circ} \mathrm{C}\right)$ deionized water was added and allowed to react. Once the sample mass stabilized, $\sim 1.5 \mathrm{~g}$ of $70 \%$ nitric acid was added to completely dissolve the remaining solid. On complete dissolution, the solution was diluted with deionized water to a mass of $50 \mathrm{~g}$. Aliquots of these original solutions were diluted with prepared aqueous $1 \%$ nitric acid to a concentration of 1-5 p.p.m. in terms of the original solid sample mass. Standard solutions were prepared for the quantification of iron and lithium in the samples. A 1 p.p.m. iron and lithium standard was prepared by diluting 1,000 p.p.m. stock solutions (SPEX CertiPrep, High-Purity Standards) with cleaned $1 \%$ nitric acid. Lower concentration standards were prepared via dilutions of the original 1 p.p.m. standard solution. Blanks of the water and acids were analysed and had negligible amounts of the analyte elements. The water used was $18 \mathrm{M} \Omega \mathrm{cm}$ (Barnstead Nanopure) and the nitric acid was purified by sub-boiling distillation (Classic Sub-boiling Still Assembly, Savillex) before use. Lithium is prone to memory effects in ICP-MS due to either sample introduction or deposition and vaporization of $\mathrm{Li}$ from the cones. The $\mathrm{Li}^{+}\left(\right.$and $\mathrm{Fe}^{+}$) signals from the samples and standards all rinsed out to baseline between the measurements.

\section{References}

1. Gatteschi, D. \& Sessoli, R. Quantum tunneling of magnetization and related phenomena in molecular materials. Angew. Chem. Int. Ed. Engl. 42, 268-297 (2003).

2. Paulsen, C., Park, J. G., Barbara, B., Sessoli, R. \& Caneschi, A. Novel features in the relaxation times of $\mathrm{Mn}_{12}$ Ac. J. Magn. Magn. Mater. 140-144, 379-380 (1995).

3. Takahashi, S. et al. Decoherence in crystals of quantum molecular magnets. Nature 476, 76-79 (2011).

4. Leuenberger, M. N. \& Loss, D. Quantum computing in molecular magnets. Nature 410, 789-793 (2001).

5. Bogani, L. \& Wernsdorfer, W. Molecular spintronics using single-molecule magnets. Nat. Mater. 7, 179-186 (2008).

6. Strandberg, T. O., Canali, C. M. \& MacDonald, A. H. Transition-metal dimers and physical limits on magnetic anisotropy. Nat. Mater. 6, 648-651 (2007).

7. Gambardella, P. et al. Giant magnetic anisotropy of single cobalt atoms and nanoparticles. Science 300, 1130-1133 (2003).

8. Khajetoorians, A. A. et al. Current-driven spin dynamics of artificially constructed quantum magnets. Science 339, 55-59 (2013).

9. Sessoli, R., Gatteschi, D., Caneschi, A. \& Novak, M. A. Magnetic bistability in a metal-ion cluster. Nature 365, 141-143 (1993).

10. Lin, P. H. et al. A polynuclear lanthanide single-molecule magnet with a record anisotropic barrier. Angew. Chem. Int. Ed. Engl. 48, 9489-9492 (2009).

11. Rinehart, J. D., Fang, M., Evans, W. J. \& Long, J. R. Strong exchange and magnetic blocking in $\mathrm{N}_{2}{ }^{3-}$-radical-bridged lanthanide complexes. Nat. Chem. 3, 538-542 (2011).

12. Osa, S. et al. A tetranuclear $3 \mathrm{~d}-4 \mathrm{f}$ single molecule magnet: $\left[\mathrm{Cu}^{\mathrm{II}} \mathrm{LTb}^{\mathrm{III}}(\mathrm{hfac})_{2}\right]_{2}$ J. Am. Chem. Soc. 126, 420-421 (2004).

13. Ishikawa, N., Sugita, M., Ishikawa, T., Koshihara, S. \& Kaizu, Y. Lanthanide double-decker complexes functioning as magnets at the single-molecular level. J. Am. Chem. Soc. 125, 8694-8695 (2003).

14. Jiang, S. D., Wang, B. W., Su, G., Wang, Z. M. \& Gao, S. A mononuclear dysprosium complex featuring single-molecule-magnet behavior. Angew. Chem. Int. Ed. Engl. 122, 7610-7613 (2010).

15. Ishikawa, N. Single molecule magnet with single lanthanide ion. Polyhedron 26, 2147-2153 (2007).

16. Luzon, J. \& Sessoli, R. Lanthanides in molecular magnetism: so fascinating, so challenging. Dalton Trans. 41, 13556-13567 (2012).

17. Freedman, D. E. et al. Slow magnetic relaxation in a high-spin iron(II) complex. J. Am. Chem. Soc. 132, 1224-1225 (2010).

18. Zadrozny, J. M. \& Long, J. R. Slow magnetic relaxation at zero field in the tetrahedral complex $\left[\mathrm{Co}(\mathrm{SPh})_{4}\right]^{2-}$. J. Am. Chem. Soc. 133, 20732-20734 (2011)

19. Zadrozny, J. M. et al. Magnetic blocking in a linear iron(I) complex. Nat. Chem. 5, 577-581 (2013).

20. Honda, K. \& Kaya, S. On the magnetization of single crystals of iron. Sci. Rep. Tohoku Univ. 15, 721-753 (1926).

21. Kaya, S. On the magnetization of single crystals of nickel, on the magnetization of single crystals of cobalt. Sci. Rep. Tohoku Univ. 17, 639-663 and 1157-1177 (1928). 
22. Klatyk, J. et al. Large orbital moments and internal magnetic fields in lithium nitridoferrate(I). Phys. Rev. Lett. 88, 207202 (2002).

23. Ksenofontov, V. et al. In situ-High Temperature Mössbauer Spectroscopy of Iron Nitrides and Nitridoferrates. Z. Anorg. Allg. Chem. 629, 1787-1794 (2003).

24. Novák, P. \& Wagner, F. R. Electronic structure of lithium nitridoferrate: effects of correlation and spin-orbit coupling. Phys. Rev. B 66, 184434 (2002).

25. Uehara, M., Barbara, B., Dieny, B. \& Stamp, P. C. E. Staircase behaviour in the magnetization reversal of a chemically disordered magnet at low temperature. Phys. Lett. A 114, 23-26 (1986).

26. Giraud, R., Tkachuk, A. M. \& Barbara, B. Quantum dynamics of atomic magnets: cotunneling and dipolar-biased tunneling. Phys. Rev. Lett. 91, 257204 (2003).

27. Rabenau, A. \& Schulz, H. Re-evaluation of the lithium nitride structure. J. Less Common Met. 50, 155-159 (1976).

28. Klatyk, J. \& Kniep, R. Crystal structure of dilithium (nitridolithiate/ferrate(I)), $\mathrm{Li}_{2}\left[\left(\mathrm{Li}_{1-}{ }_{x} \mathrm{Fe}_{x}\right) \mathrm{N}\right], x=0.63$. Z. Krist. New Cryst. Struct. 214, 447-448 (1999).

29. Yamada, A., Matsumoto, S. \& Nakamura, Y. Direct solid-state synthesis and large-capacity anode operation of $\mathrm{Li}_{3-} \mathrm{Fe}_{x} \mathrm{~N}$. J. Mater. Chem. 21, 10021-10025 (2011).

30. Gregory, D. H. Nitride chemistry of the s-block elements. Coord. Chem. Rev 215, 301-345 (2001).

31. Barbara, B. et al. Evidence for resonant magnetic tunneling of rare-earth ions: from insulating to metallic matrix. J. Magn. Magn. Mater. 272-276, 1024-1029 (2004).

32. Wernsdorfer, W. \& Sessoli, R. Quantum phase interference and parity effects in magnetic molecular clusters. Science 284, 133-135 (1999).

33. Cullity, B. D. in Introduction to Magnetic Materials. (ed. Cohen, M.) 442 (Addison-Wesley Publishing Company Reading, 1972).

34. Sangregorio, C., Ohm, T., Paulsen, C., Sessoli, R. \& Gatteschi, D. Quantum tunneling of the magnetization in an iron cluster nanomagnet. Phys. Rev. Lett. 78, 4645-4648 (1997).

35. Giraud, R., Wernsdorfer, W., Tkachuk, A. M., Mailly, D. \& Barbara, B. Nuclear spin driven quantum relaxation in $\mathrm{LiY}_{0.998} \mathrm{Ho}_{0.002} \mathrm{~F}_{4}$. Phys. Rev. Lett. 87, 057203 (2001).

36. Ishikawa, N., Sugita, M., Ishikawa, T., Koshihara, S. \& Kaizu, Y. Mononuclear lanthanide complexes with a long magnetization relaxation time at high temperatures: a new category of magnets at the single-molecular level. J. Phys. Chem. B 108, 11265-11271 (2004).

37. Gonidec, M. et al. Surface supramolecular organization of a terbium(III) double-decker complex on graphite and its single molecule magnet behavior. J. Am. Chem. Soc. 133, 6603-6612 (2011)

38. Wernsdorfer, W., Aliaga-Alcalde, N., Hendrickson, D. N. \& Christou, G. Exchange-biased quantum tunnelling in a supramolecular dimer of singlemolecule magnets. Nature 416, 406-409 (2002).
39. Kirschbaum, K., Martin, A., Parrish, D. A. \& Pinkerton, A. A. Cooperative Jahn-Teller induced phase transition of $\mathrm{TbVO}_{4}$ : single crystal structure analyses of the tetragonal high temperature phase and the twinned orthorhombic phase below 33 K. J. Phys. Condens. Matter 11, 4483 (1999).

40. Jahn, H. A. \& Teller, E. Stability of polyatomic molecules in degenerate electronic states. I. orbital degeneracy. Proc. R Soc. Lond. A 161, 220-235 (1937).

41. Canfield, P. C. \& Fisher, I. R. High-temperature solution growth of intermetallic single crystals and quasicrystals. J. Cryst. Growth 225, 155-161 (2001).

\section{Acknowledgements}

Bruce Harmon, Yongbin Lee, Natalia Perkins, Yuriy Sizyuk, Vladimir Antropov, Makariy Tanatar, Hyunsoo Kim, Ruslan Prozorov and Yuji Furukawa are acknowledged for comments and discussions. The authors thank Gregory Tucker for assistance with recording Laue-back-reflection pattern, Jakoah Brgoch for assistance with early X-ray powder diffraction measurements and Jim Anderegg for discussions and first attempts of performing Auger spectroscopy on these samples. Kevin Dennis is acknowledged for assistance with magnetization measurements. This work was supported by the US Department of Energy, Office of Basic Energy Science, Division of Materials Sciences and Engineering. The research was performed at the Ames Laboratory. Ames Laboratory is operated for the US Department of Energy by Iowa State University under Contract No. DE-AC02-07CH11358.

\section{Author contributions}

A.J. and P.C.C. developed the $\mathrm{Li}-\mathrm{N}$ growth technique and initiated this study. A.J. grew the single crystals and performed the magnetization measurements. S.T. collected and analysed single crystal X-ray diffraction data. J.L.J. and R.S.H. performed the chemical analysis. P.C.C., R.W.M., S.L.B., V.T. and A.J. analysed and interpreted the magnetization data. A.K. and A.J. collected and analysed powder X-ray and Laue-back-reflection data A.J. and P.C.C. wrote the manuscript with the help of all authors.

\section{Additional information}

Supplementary Information accompanies this paper at http://www.nature.com/ naturecommunications

Competing financial interests: The authors declare no competing financial interests.

Reprints and permission information is available online at http://npg.nature.com/ reprintsandpermissions/

How to cite this article: Jesche, A. et al. Giant magnetic anisotropy and tunnelling of the magnetization in $\mathrm{Li}_{2}\left(\mathrm{Li}_{1-x} \mathrm{Fe}_{x}\right) \mathrm{N}$. Nat. Commun. 5:3333 doi: 10.1038/ncomms4333 (2014) 